\title{
Differential approach to peripheral blood cell ratios in patients with systemic lupus erythematosus and various manifestations
}

\author{
Dorota Suszek $^{1}$ (D) - Anna Górak ${ }^{1}$ (D) - Maria Majdan ${ }^{1}$ (D)
}

Received: 28 April 2020 / Accepted: 28 July 2020 / Published online: 9 August 2020

(c) The Author(s) 2020

\begin{abstract}
New markers of systemic lupus erythematosus (SLE) activity are under investigation. In recent years, the researchers have been focusing increased attention on the role of haematological indicators in assessing the disease activity. Specifically, neutrophil-, basophil-, eosinophil-, monocyte- and platelet-to-lymphocyte ratios (NLR, BLR, ELR, MLR and PLR) have been considered. The specific objective of this study was to determine the suitability of the haematological markers for the assessment of SLE activity and SLE-related organ damage. This study is a retrospective analysis of 136 patients with SLE (124 women and 12 men) who received chloroquine/hydroxychloroquine (HQ/HCQ) monotherapy or HQ/HCQ therapy combined with low/medium doses of glucocorticoid. All patients were assessed for disease activity using the Systemic Lupus Erythematosus Disease Activity Index 2000 (SLEDAI-2K) scale. In addition, erythrocyte sedimentation rate (ESR) and C-reactive protein (CRP) inflammatory parameters were determined in each patient. NLR, BLR, ELR, MLR and PLR were evaluated and correlated with the SLE activity parameters and inflammatory markers. The mean values of the haematological indicators were compared in particular manifestations of SLE-induced organ damage. For numerical variables, descriptive statistics were calculated: median, standard deviation, minimum and maximum values. The Mann-Whitney $U$ test was used for the comparison of continuous variables in the two groups. The Spearman rank correlation test was used to search for any relationships between variables. A $p$ value $<0.05$ was considered to be statistically significant. We have found a positive correlation between ELR, MLR and the SLEDAI scale ( $r=0.22$ and $r=0.27$, respectively). NLR, MLR and PLR ratios were significantly correlated with ESR and CRP. Considerably higher NLR values were found in patients with cutaneous and/or mucosal symptoms and with kidney involvement compared to patients without such involvement $(4.26 \pm 4.2$ vs $3.27 \pm 2.7$; $p=0.05$ and $5.45 \pm 5.6$ vs $3.05 \pm 2.0 ; p<0.001$ respectively). BLR and MLR were significantly higher in patients manifesting symptoms of vasculitis $(0.09 \pm 0.1$ vs $0.02 \pm 0.01 ; p<0.001$ and $3.1 \pm 4.2$ vs $0.3 \pm 0.1 ; p<0.001$ respectively), arthritis and/or myositis $(0.04 \pm 0.09$ vs $0.02 \pm 0.01 ; p=0.01$ and $1.02 \pm 2.6$ vs $0.35 \pm 0.4 ; p=0.01$ respectively), whereas elevated ELR ratios were observed in patients with vasculitis $(0.4 \pm 0.5$ vs $0.08 \pm 0.06 ; p<0.001)$ compared to patients without such organ involvement. The PLR marker was substantially higher in patients exhibiting haematological disorders in the course of SLE $(276.6 \pm 226.4$ vs $192.6 \pm 133.5 ; p=0.01)$. The results indicate that ELR and MLR are effective markers of SLE activity. The haematological indicators may predict SLE-dependent organ damage, particularly cutaneous, mucosal, arthritic, myositic, haematological and kidney involvement.
\end{abstract}

Keywords Lupus erythematosus $\cdot$ Haematological markers $\cdot$ Disease activity

\section{Introduction}

Systemic lupus erythematosus (SLE) is a chronic autoimmune disease that leads to inflammation of multiple tissues and organs. The constitutional symptoms of SLE predominantly involve damage to the skin, joints, kidneys, the central nervous system and bone marrow [1]. The diagnosis of the disease was based on the 2012 Systemic Lupus International Collaborating Clinics (SLICC) qualification criteria and the 
2019 EULAR/ACR criteria [2, 3]. SLE treatment depends on the disease activity as well as the type of organs involved. In clinical practice, the assessment of disease activity in SLE is performed by means of disease activity scores Systemic Lupus Erythematosus Disease Activity Index 2000 (SLEDAI-2K) or additional testing for the complement components 3 and $4(C 3, C 4)$ and anti-dsDNA titers [4]. The search for new markers of SLE activity is ongoing [5, 6]. In recent years, there has been a surge of interest in the role of haematological indicators in the assessment of SLE activity. Neutrophil-, basophil-, eosinophil-, monocyte- and platelet-to-lymphocyte ratios (NLR, BLR, ELR, MLR and PLR) have been found to indirectly reflect subclinical inflammation [7]. Their value for assessing or projecting the disease activity has been established in autoimmune diseases, such as primary Sjögren's syndrome (pSS), psoriasis, systemic vasculitis, ulcerative colitis as well as in cancer and infectious diseases [8-15].

\section{Objectives}

The objective of this study was to determine the usefulness of haematological indicators in the assessment of SLE activity and organ involvement.

\section{Patients and methods}

A total of 136 patients with SLE (124 women and 12 men) aged $40.1 \pm 14$ years (22-74) hospitalised in the Clinic of Rheumatology and Connective Tissue Diseases at the Medical University of Lublin between 2012 and 2018 were enroled in the present retrospective study. All patients conformed with the 2012 SLICC criteria for SLE. Cutaneous and mucosal symptoms were present in 26 patients (19.1\%), arthritis in 13 patients (9.5\%), lupus nephritis in 23 patients (16.9\%), haematological symptoms in $26(19.1 \%)$ and vasculitis was manifested in 5 (3.7\%). Two patients exhibited signs of damage to the nervous system and serositis. With respect to treatment, the patients received chloroquine/hydroxychloroquine (HQ/HCQ) monotherapy or $\mathrm{HQ} / \mathrm{HCQ}$ therapy combined with low/medium doses of glucocorticoids (prednisone $6.3 \mathrm{mg} /$ per day; $2.5-10 \mathrm{mg}$ ). Patients treated with high doses of glucocorticoids and strong immunosuppressants, overlap syndrome, infections, cancer, end-stage renal failure, diseases of the liver or the haematopoietic system were excluded from the study group.

The patients were evaluated for disease activity according to the SLEDAI-2K scale. In each patient, NLR, BLR, ELR, MLR and PLR indicators were determined (Table 1). The haematological markers were subsequently correlated with disease activity parameters, C-reactive protein (CRP) and
Table 1 The haematological indicators and disease activity parameters in systemic lupus erythematosus patients

\begin{tabular}{lc}
\hline $\begin{array}{l}\text { Haematological indicators and disease } \\
\text { activity parameters; } n=136\end{array}$ & Median value (min.-max.) \\
\hline CRP (mg/dl) (range 0-5) & $8.1(0-107)$ \\
ESR (mm/h) & $23.4(0.12-109)$ \\
C3 (mg/dl) (range 85-160) & $91.7(11.4-151.8)$ \\
SLEDAI-2K & $3.5(2-22)$ \\
Anti-dsDNA (\% positive) & $101 / 136(74 \%)$ \\
NLR & $3.46(0.6-22.2)$ \\
BLR & $0.02(0-0.4)$ \\
ELR & $0.09(0-1.3)$ \\
MLR & $0.4(0.1-9.9)$ \\
PLR & $208.6(18-1090)$ \\
\hline
\end{tabular}

anti-dsDNA anti-dsDNA antibodies, $C 3$ the complement component 3, CRP C-reactive protein, ESR erythrocyte sedimentation rate, $B L R$ basophil-to-lymphocyte ratio, ELR eosinophil-to-lymphocyte ratio, $M L R$ monocyte-to-lymphocyte ratio, $N R L$ neutrophil-to-lymphocyte ratio, $P L R$ platelet-to-lymphocyte ratio, SLEDAI- $K$ Systemic Lupus Erythematosus Disease Activity Index 2000

erythrocyte sedimentation rate (ESR). The average values of the markers were compared across individual SLE-induced organ damage manifestations (Table 2).

\section{Statistical analysis}

The analysis was carried out using STATISTICA 10 software. For numerical variables, descriptive statistics were calculated: median, minimum and maximum values. The Mann-Whitney U test was used for comparison of continuous variables in the two groups (with/without examined SLE symptoms). The Spearman rank correlation test was used to search for any relationships between variables. A $p$ value $<0.05$ was considered to be statistically significant.

\section{Results}

In Table 1, the examined haematological indicators and disease activity in SLE patients are presented.

The tests have revealed a positive correlation between ELR, MLR and the SLEDAI scale ( $r=0.22$ and $r=0.27$, respectively). NLR, MLR and PLR ratios were significantly correlated with ESR $(r=0.24 ; r=0.33 ; r=0.2$, respectively) (Figs. 1 and 2) and PLR with CRP $(r=0.2)$.

Table 2 compares the average concentrations of haematological indicators: NLR, BLR, ELR, MLR and PLR in patients with SLE with various manifestations of organ disease. The values of these indicators were not compared in patients presenting serositis and neurological symptoms due to the inadequate number of patients ( 2 patients) manifesting 
Table 2 A comparison of NLR, BLR, ELR, MLR and PLR concentrations in systemic lupus erythematosus patients in various manifestations of organ damage

\begin{tabular}{|c|c|c|c|c|c|c|}
\hline Symptoms of SLE & NLR & BLR & ELR & MLR & PLR & $p$ \\
\hline \multicolumn{7}{|c|}{ Cutaneous and/or mucosal } \\
\hline (+) $n=26$ & $4.26(0.8-22.2)^{*}$ & $0.025(0-0.1)$ & $0.1(0-0.7)$ & $0.51(0.1-5.0)$ & $236.5(81-890.4)$ & \multirow[t]{2}{*}{$p=0.05^{*}$} \\
\hline$(-) n=110$ & $3.27(0.6-21.4)$ & $0.022(0-0.4)$ & $0.09(0-1.3)$ & $0.39(0.1-9.9)$ & $202.08(18-1090)$ & \\
\hline \multicolumn{6}{|l|}{ Vasculitis } & \multirow{3}{*}{$\begin{array}{l}p<0.001 * \\
p<0.001 * * \\
p<0.001 * * *\end{array}$} \\
\hline$(+) n=5$ & $3.09(1.2-4.4)$ & $0.09(0-0.4)^{*}$ & $0.4(0-1,3)^{* *}$ & $3.1(0.1-9.9)^{* * *}$ & $199.68(123.5-314.7)$ & \\
\hline (-) $n=131$ & $3.47(0.6-22.2)$ & $0.02(0-0.1)$ & $0.08(0-0.3)$ & $0.3(0-1.5)$ & $209.01(18-1090)$ & \\
\hline \multicolumn{7}{|c|}{ Arthritis and/or myositis } \\
\hline$(+) n=13$ & $2.76(1.2-6.6)$ & $0.04(0-0.4)^{*}$ & $0.15(0-1.3)$ & $1.02(0.1-9.9)^{* *}$ & $215.9(70.6-679.4)$ & $p=0.01^{*}$ \\
\hline (-) $n=123$ & $3.53(0.6-22.2)$ & $0.02(0-0.1)$ & $0.09(0-0.7)$ & $0.35(0.1-5.0)$ & $207.9(18-1090)$ & $p=0.01 * *$ \\
\hline \multicolumn{7}{|l|}{ Nephritis } \\
\hline (+) $n=23$ & $5.45(0.6-22.2)^{*}$ & $0.03(0-0.1)$ & $0.12(0-0.7)$ & $0.56(0.1-5)$ & $271.3(41.8-890.5)^{* *}$ & \multirow{2}{*}{$\begin{array}{l}p<0.001 * \\
p=0.03 * *\end{array}$} \\
\hline$(-) n=113$ & $3.05(0.9-13.6)$ & $0.02(0-0.4)$ & $0.09(0-1.3)$ & $0.38(0.1-9.9)$ & $195.9(18-890.5)$ & \\
\hline \multicolumn{7}{|l|}{ Haematological } \\
\hline (+) $n=26$ & $4.1(0.6-22.2)$ & $0.02(0-0.1)$ & $0.07(0-0.3)$ & $0.3(0.1-1.5)$ & $276.6(41.8-1090)^{*}$ & \multirow[t]{2}{*}{$p=0.01 *$} \\
\hline (-) $n=110$ & $3.3(0.8-21.4)$ & $0.02(0-0.4)$ & $0.1(0-1.3)$ & $0.4(0.1-9.9)$ & $192.6(18-890.5)$ & \\
\hline \multicolumn{7}{|l|}{ Anti-dsDNA } \\
\hline (+) $n=101$ & $3.7(0.6-22.2)^{*}$ & $0.02(0-0.1)$ & $0.09(0-0.7)$ & $0.36(0.1-5)$ & $214.7(41.8-890.5)$ & \multirow[t]{2}{*}{$p=0.03 *$} \\
\hline (-) $n=35$ & $2.8(1.1-9.6)$ & $0.03(0-0.4)$ & $0.11(0-1.3)$ & $0.58(0.1-9.9)$ & $191.3(18-1090)$ & \\
\hline
\end{tabular}

anti-dsDNA anti-dsDNA antibodies, $B L R$ basophil-to-lymphocyte ratio, $E L R$ eosinophil-to-lymphocyte ratio, $M L R$ monocyte-to-lymphocyte ratio, $N R L$ neutrophil-to-lymphocyte ratio, $P L R$ platelet-to-lymphocyte ratio

$*, * *, * * *$ indicates statistically significant differences between two values

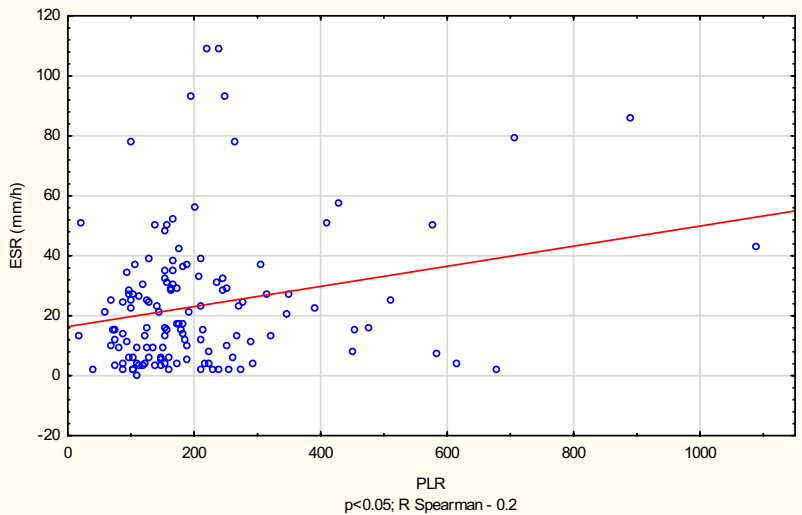

Fig. 1 Correlation between erythrocyte sedimentation rate (ESR) and platelet-to-lymphocyte ratio (PLR)

the symptoms. Considerably higher NLR values were found in patients with cutaneous and/or mucosal symptoms and with kidney involvement, compared to patients without such involvement. BLR and MLR were significantly higher in patients manifesting symptoms of vasculitis, arthritis and/ or myositis, whereas elevated ELR ratios were observed in patients with vasculitis compared to patients without such organ involvement. The PLR marker was substantially higher in patients exhibiting haematological disorders in the course of SLE.

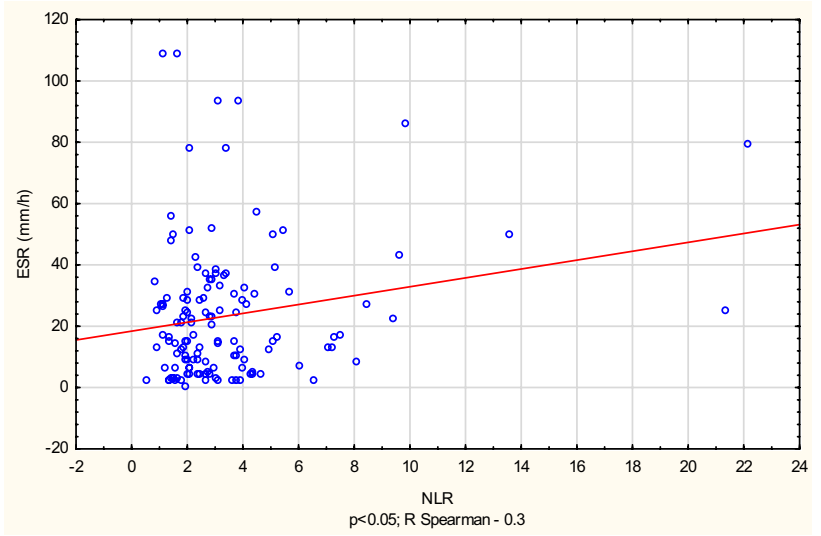

Fig. 2 Correlation between erythrocyte sedimentation rate (ESR) and neutrophil-to-lymphocyte ratio (NLR)

\section{Discussion}

In recent years, there has been a growing emphasis on early diagnosis of SLE exacerbation and monitoring of SLE activity. Simultaneously, concentrated efforts have been made to search for markers capable of prognosing SLE exacerbation in the preclinical period, which could, furthermore, indicate signs of exacerbation in a given organ. A considerable literature has provided evidence 
for the efficacy of haematological indicators, i.e. NLR, BLR, ELR, MLR and PLR in monitoring the activity of a number of inflammatory joint diseases, including SLE, rheumatoid arthritis (RA), pSS or systemic sclerosis. What is more, NLR has been proved to successfully predict the development of lupus nephritis (LN).

From the results of our study, it can be seen that ELR and MLR significantly correlated with the SLEDAI scale, whereas NLR and PLR exhibited no relationship with the disease activity markers. There was a significant positive correlation between NLR/MLR/PLR indicators and ESR/ CRP values. With respect to organ involvement, significantly higher NLR was observed in patients with cutaneous and/ or mucosal symptoms and kidney damage. Moreover, NLR was also elevated in patients with anti-dsDNA antibodies. BLR and MLR were significantly higher in patients manifesting symptoms of vasculitis, arthritis and/or myositis, while ELR - in patients with vasculitis. Significantly higher PLR rates were recorded in patients with haematological manifestations of SLE. ELR and BLR demonstrated a good capacity for assessing SLE activity. Considering the prognosis of organ involvement, there were NLR, MLR, BLR and ELR that showed good performance in the assessment of cutaneous lesion, NLR and PLR in the prediction of renal damage, whereas BLR and MLR showed good correlation with arthritic manifestations and PLR with haematological symptoms.

Waffa et al. observed a significant correlation between NLR/PLR and the SLEDAI scale and C4. Moreover, in the course of their study, NLR was found to be a good marker of renal function parameters, proteinuria, anti-dsDNA and histopathological changes in kidney biopsy. Both NLR and PLR exhibited sufficient accuracy in the prediction of kidney damage in SLE. What is more, the haematological markers correlated well with the inflammation markers ESR and CRP [9]. Similar findings were presented by Qin et al. [16]. In contrast to earlier findings, in our study, the relationship was observed between NLR and ESR.

$\mathrm{Wu}$ et al. reported elevated levels of NLR and PLR in patients with active SLE. The neutrophil-to-lymphocyte ratio was significantly higher in patients with kidney involvement and was, furthermore, an accurate marker of SLE exacerbation [17]. A similar relationship in SLE patients has been confirmed in earlier studies: Soliman et al., Li et al. and Ayna et al. [18-20]. While confirming the predictive value of NLR in assessing SLE activity, Yu et al. determined a new indicator- $\mathrm{NC} 3 \mathrm{R}$-neutrophil-to-C3 ratio [21]. In a far more extensive study, Yang et al. examined 1139 patients presenting a range of inflammatory arthritic diseases (SLE, RA, pSS, dermatomyositis, polymyositis, mixed connective tissue disease, rheumatic polymyalgia and ankylosing spondylitis) and osteoarthritis (OA). From their findings, it can be seen that notably higher NLR and MLR ratios were prevalent in patients with inflammatory arthritic diseases. In the majority of patients, significantly lower BLR was observed. Compared with other inflammatory arthritic diseases, lower ELR was recorded in patients with SLE [22]. Ma et al. and Wang et al. performed meta-analysis to investigate the relationship between NLR, PLR and SLE. Fourteen studies with 1246 SLE patients (Ma et al.) and 1781 SLE patients (Wang et al.) were included in this meta-analysis. The meta-analysis demonstrated elevated levels of NLR and PLR in patients with active SLE (Ma et al.) and positive clinical value of NRL for diagnosing SLE, active SLE or LN (Wang et al.) [23, 24].

Despite SLE activity scores being widely available for clinicians, haematological indicators constitute a considerably faster and more easily accessible biomarker of the disease activity. In the available literature, there is only one study that assesses the predictive value of all five haematological indicators (NLR, MLR, BLR, ELR and PLR) in monitoring SLE activity. In earlier works, however, the markers were not measured in correlation with organ manifestations of SLE, with the exception of patients with lupus nephritis. Although there are limitations due to a relatively small group of patients, the retrospective analysis approach or short-term observation, we are inclined to believe that these cost-effective and widely available markers could constitute a positive addition to a daily clinical practice involving the assessment of SLE activity.

In conclusion, the haematological indicators may predict SLE-dependent organ damage, particularly cutaneous, mucosal, arthritic, myositic, haematological and kidney involvement. Future studies should account for a comparative analysis of the indicators in question in various systemic diseases.

Open Access This article is licensed under a Creative Commons Attribution 4.0 International License, which permits use, sharing, adaptation, distribution and reproduction in any medium or format, as long as you give appropriate credit to the original author(s) and the source, provide a link to the Creative Commons licence, and indicate if changes were made. The images or other third party material in this article are included in the article's Creative Commons licence, unless indicated otherwise in a credit line to the material. If material is not included in the article's Creative Commons licence and your intended use is not permitted by statutory regulation or exceeds the permitted use, you will need to obtain permission directly from the copyright holder. To view a copy of this licence, visit http://creativecommons.org/licenses/by/4.0/.

\section{References}

1. Jeleniewicz R, Suszek D, Majdan M (2015) Clinical picture of late-onset systemic lupus erythematosus in a group of Polish patients. Pol Arch Med Wewn 125(7-8):538-544. https://www. mp.pl/paim/issue/article/2963 
2. Petri M, Orbai A-M, Alarcón GS, Gordon C et al (2012) Derivation and validation of Systemic Lupus International Collaborating Clinics (SLICC) classification criteria for systemic lupus erythematosus. Arthritis Rheum 64:2677-2686. https://doi.org/10.1002/ $\operatorname{art.34473}$

3. Aringer M, Costenbader K, Daikh D, Brinks R, Mosca M, Ramsey-Goldman R et al (2019) 2019 European league against Rheumatism/American College of Rheumatology Classification criteria for systemic lupus erythematosus. Arthritis Rheumatol 71:1400-1412. https://doi.org/10.1002/art.40930

4. Mikdashi J, Nived O (2015) Measuring disease activity in adults with systemic lupus erythematosus: the challenges of administrative burden and responsiveness to patient concerns in clinical research. Arthritis Res Ther 17:183. https://doi.org/10.1186/s1307 5-015-0702-6

5. Winthrop KL, Weinblatt ME, Bathon J, Burmester GR (2020) Unmet need in rheumatology: reports from the Targeted Therapies meeting 2019. Ann Rheum Dis 79:88-93. https://ard.bmj.com/ content/79/1/88

6. Dörner T, Furie R (2019) Novel paradigms in systemic lupus erythematosus. Lancet 8(393):2344-2358. https://doi.org/10.1016/ S0140-6736(19)30546-X

7. Haitao Y, Lili J, Liqiong Y, Chao G et al (2018) Predictive value of the neutrophil-to-lymphocyte ratio and hemoglobin in systemic lupus erythematosus. Exp Ther Med 16(2):1547-1553. https://doi. org/10.3892/etm.2018.6309

8. Gasparyan AY, Ayvazyan L, Mukanova U, Yessirkepov M et al (2019) The platelet-to-lymphocyte ratio as an inflammatory marker in rheumatic diseases. Ann Lab Med 39(4):345-357. https ://doi.org/10.3343/alm.2019.39.4.345

9. Wafaa MS, Nahed MS, Iman MG, Mohja A (2018) Neutrophil to lymphocyte and platelet to lymphocyte ratios in systemic lupus erythematosus: relation with disease activity and lupus nephritis. Rheumatol Clin. https://doi.org/10.1016/j.reuma.2018.07.008

10. Zencir C, Akpek M, Senol S, Selvi M et al (2015) Association between hematologic parameters and in-hospital mortality in patients with infective endocarditis. Kaohsiung J Med Sci 31:632638. https://doi.org/10.1016/j.kjms.2015.10.004

11. Osadnik T, Wasilewski J, Lekston A, Strzelczyk J et al (2015) The platelet-to-lymphocyte ratio as a predictor of all-cause mortality in patients with coronary artery disease undergoing elective percutaneous coronary intervention and stent implantation. J Saudi Heart Assoc 27:144-151

12. Koh CH, Bhoo-Pathy N, Ng KL, Jabir RS et al (2015) Utility of pre-treatment neutrophil-lymphocyte ratio and platelet-lymphocyte ratio as prognostic factors in breast cancer. Br J Cancer 113:150-158. https://www.nature.com/articles/bjc2015183

13. Yang XF, Ding FM, Ye YC, Zhang SY (2018) Relationship between neutrophil-to-lymphocyte ratio and pulse wave velocity in young patients with systemic lupus erythematosus. Chin Med (Eng) 131(1):10-15. https://doi.org/10.4103/0366-6999.221272

14. Lian Z, You-You X, Chong Z (2015) Application of platelet/lymphocyte and neutrophil/lymphocyte ratios in early diagnosis and prognostic prediction in patients with resectable gastric cancer.
Cancer Biomark 15(6):899-907. https://doi.org/10.3233/cbm150534

15. Jaszczura M, Góra A, Grzywna-Rozenek E, Barć-Czarnecka M et al (2019) Analysis of neutrophil to lymphocyte ratio, platelet to lymphocyte ratio and mean platelet volume to platelet count ratio in children with acute stage of immunoglobulin A vasculitis and assessment of their suitability for predicting the course of the disease. Rheumatol International 39:869-878. https://doi. org/10.1007/s00296-019-04274-z

16. Qin B, Ma N, Tang Q, Wei T, Zhong R et al (2016) Neutrophil to lymphocyte ratio (NLR) and platelet to lymphocyte ratio (PLR) were useful markers in assessment of inflammatory response and disease activity in SLE patients. Mod Rheumatol 26(3):372-376. https://doi.org/10.3109/14397595.2015.1091136

17. Wu Y, Chen Y, Yang X, Chen L et al (2016) Neutrophil-to-lymphocyte ratio (NLR) and platelet-to- lymphocyte ratio (PLR) were associated with disease activity in patients with systemic lupus erythematosus. Int Immunopharmacol 36:94-99

18. Soliman WM, Sherif NM, Ghanima IM, El-Badawy MA (2018) Neutrophil to lymphocyte and platelet to lymphocyte ratios in systemic lupus erythematosus: relation with disease activity and lupus nephritis. Reumatol Clín. https://doi.org/10.1016/j. reuma.2018.07.008

19. Li L, Xia Y, Chen C, Cheng P et al (2015) Neutrophil-lymphocyte ratio in systemic lupus erythematosus disease: a retrospective study. Int J Clin Exp Med 8:11026

20. Ayna AB, Ermurat S, Coşkun BN, Harman H et al (2017) Neutrophil to lymphocyte ratio and mean platelet volume as inflammatory indicators in systemic lupus erythematosus nephritis. Arch Rheumatol 32:021-025. https://doi.org/10.5606/archrheuma tol.2017.5886

21. Yu J, Zeng T, Wu Y, Tian Y et al (2019) Neutrophil-to-C3 ratio and neutrophil-to-lymphocyte ratio were associated with disease activity in patients with systemic lupus erythematosus. J Clin Lab Anal. https://doi.org/10.1002/jcla.22633

22. Yang Z, Zhang Z, Lin F, Ren Y et al (2017) Comparisons of neutrophil-, monocyte-, eosinophil-, and basophil- lymphocyte ratios among various systemic autoimmune rheumatic diseases. APMIS 125(10):863-871. https://doi.org/10.1111/apm.12722

23. Ma L, Zeng A, Chen B, Chen Y, Zhou R (2019) Neutrophil to lymphocyte ratio and platelet to lymphocyte ratio in patients with systemic lupus erythematosus and their correlation with activity: a meta-analysis. Int Immunopharmacol 76:105949. https://doi. org/10.1016/j.intimp.2019.105949

24. Wang L, Wang C, Jia X, Yang M, Yu J (2020) Relationship between neutrophil-to-lymphocyte ratio and systemic lupus erythematosus: a meta-analysis. Clinics (Sao Paulo) 75:e1450. https ://doi.org/10.6061/clinics/2020/e1450

Publisher's Note Springer Nature remains neutral with regard to jurisdictional claims in published maps and institutional affiliations. 